\title{
"Real Baby - Real Family"- Holdable tangible baby VR
}

\author{
Yuya MOCHIZUKI \\ Kanagawa Institute of Technology \\ baby@shirai.la \\ Takaya ASANO \\ Kanagawa Institute of Technology
}

\author{
Rex HSIEH \\ Kanagawa Institute of Technology \\ Marika HIGASHIDA \\ Kanagawa Institute of Technology \\ Akihiko SHIRAI \\ Kanagawa Institute of Technology
}

\author{
Daiki AGATSUMA \\ Kanagawa Institute of Technology \\ Tatsuya NISHIKIZAWA \\ Kanagawa Institute of Technology
}

\begin{abstract}
This project "Real Baby - Real Family" is a project aimed at expressing love and family ties utilizing Virtual Reality system (VR). In this paper, we described the version presented at the International collegiate Virtual Reality Contest (IVRC 2016). It reports system design and implementation, user evaluation during exhibition, and future possibilities. The single player version of "Real Baby" exhibited at the IVRC 2016 preview was made up of three main elements. The first element is a holdable baby mock-up without using HMD. The second is a generator of baby face based on a user's 2D facial image. The third elements consists of a visual, vocal, and haptic feedback as well as event generation. In the future, we are hoping to examine alternative expressions of "love in family ties" with this project. Supplementary materials related to this article can be found online at "https://youtu.be/Gy_XrWfl4RE"
\end{abstract}

\section{CCS CONCEPTS}

-Software and its engineering $\rightarrow$ Virtual worlds training simulations;

\section{KEYWORDS}

VR, Baby, Face Generation, Haptic Feedback, Nursing Simulator, Entertainment VR

\section{ACM Reference format:}

Yuya MOCHIZUKI, Rex HSIEH, Daiki AGATSUMA, Takaya ASANO, Marika HIGASHIDA, Tatsuya NISHIKIZAWA, and Akihiko SHIRAI. 2017. "Real Baby - Real Family”- Holdable tangible baby VR. In Proceedings of VRIC '17, Laval, France, March 22-24, 2017, 4 pages.

DOI: $10.1145 / 3110292.3110297$

\section{MOTIVATION}

Babies are the fruits of parental love. Such "conjugal love" and "love in family ties" can be used as a synonym for a child, or a baby. This project, named "Real Baby - Real Family"[7], is a virtual reality

Permission to make digital or hard copies of all or part of this work for personal or classroom use is granted without fee provided that copies are not made or distributed for profit or commercial advantage and that copies bear this notice and the full citation on the first page. Copyrights for components of this work owned by others than the author(s) must be honored. Abstracting with credit is permitted. To copy otherwise, or republish, to post on servers or to redistribute to lists, requires prior specific permission and/or a fee. Request permissions from permissions@acm.org.

VRIC '17, Laval, France

(C) 2017 Copyright held by the owner/author(s). Publication rights licensed to ACM

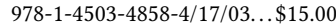

DOI: $10.1145 / 3110292.3110297$ project that allows friends, homosexual couples, and heterosexual couples to experience the joy of raising a child. It is our hope to invoke deep discussions about love with this VR project. In this paper, we report the concept of this project during the 24th International Collegiate Virtual Reality Contest (IVRC 2016). The system was presented at the Tokyo qualification and later finals competition. We also discussed the reactions of players and future possibilities with this project.

\section{RELATED WORKS}

Below are some of the previous researches that also uses baby as their subject matter. Neuro-Baby [10],Infanoid [3], Kismet [5], Cog [9], Babybot [6], YOTARO [8]. Amongst these researches YOTARO can be considered as a work that manifests a realistic baby. YOTARO is a baby robot that has a runny nose and many different emotions. However, because YOTARO's face is that of an illustration and not a real human being, it looks as if the baby is not related to the players. Our project generates a baby from facial images of the heterosexual or homosexual couple playing it thus those experiencing this project get a baby looking just like the players. While YOTARO can be considered as a precedent that represents a realistic VR baby robot, its face which is composed of an illustration makes it look like a fictional character or a baby belonging to another person. Another notable work worth mentioning is "(Im)possible_baby"[2] which was awarded the Excellence Award at the 19th Japan Media Arts Festival. This work makes people guess if the child resulted from genetic information of a real couple or produced from a family photo. While it is possible to produce a baby face image from homosexual or heterosexual couples with this work, it lacks real-time and interactive components. According to the "Facial aging simulation based on facial domain knowledge" [4] of Isono, by combining the averaged face and an actual childhood photo, the removal of wrinkles and age spots while keeping the characteristics of the player was possible. After going through further treatments to smoothen the skin area, it is possible to generate age reduction images.

\section{DESIGN}

This Virtual Reality system is characterized by a virtual family enabling the players to communicate with a baby through haptic, visual, and audio feedbacks while wearing a head-mounted display (HMD). According to our hypothesis, creating a believable baby looking like our players will make people more emotionally invested with the experience. Thus we created a baby generated 
from players' photographs. The most important part of our project; however, is that we constructed a physical baby that can interact with the audience. Projects such as YOTARO features a lot of interactivity but none allows the player to interact with the baby through haptic means. This means one cannot hug the baby which is a fundamental interaction in raising a baby. On the other hand, projects such as Laerdal's high performance infant medical training simulator "SimBaby" ${ }^{\mathrm{TM}}$ " and "SimNewB ${ }^{\circledR}$ " [1] focuses on treatment and is too expensive for the general public. The biggest difference between baby simulator in the medical and VR world is those developed for medical purpose aims at patient treatment while VR baby simulator aims at creating a believable experience that touches the hearts of the players. Real Baby - Real Family is unique for allowing players to (1) hug and interact with the baby physically, (2) creating a baby looking just like the players, (3) contains audio components.

\subsection{Holdable baby device}

In order to hug a baby doll without experiencing discomfort while wearing the HMD, there is a need for high precision position tracking of the baby doll. However, this is hindered by image recognition ability of a one eyed camera therefore slowing down the position tracking speed. Our proposed method uses multipoint image sensor implemented in the controller of HTC Vive to enable high precision position tracking. This allows players to hug the baby without getting visually disoriented (fig.1).

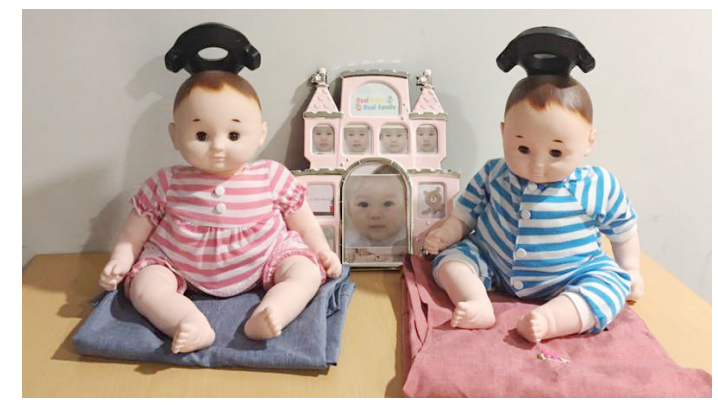

Figure 1: HTC Vive and Baby mock-up

\subsection{Visual Face Generator}

Below are three characteristics of our baby face generator: (1) Generating a face fitting the players' skin color, (2) Creating the baby's face from multiple photographs, (3) Reversing the age of the players to fit that of the baby.

3.2.1 Average Baby Face Generation. It determines the skin color of the baby by averaging the skin tone of the two photographs. Our system also morphs the colors and shapes of the faces using OpenCV. Lastly it collects 16 baby face images using Japanese Google Image Search.

3.2.2 Get Face Landmark Index. In order to morph the many photographs, baby images, and corresponding points in the feature points, it is necessary to obtain the index. In the proposed method, we use the Dlib of open source library, with results that have been learned by the data set iBUG-300-W, to get the points of each part of the face. As a result of fig.2, the eyes, noses, mouths, eyebrows, the feature points, and the 68 points with index composed of contour, are realized by automation morphing. Here it can be seen that morphing are concentrated in the mouth and even parts of the face.

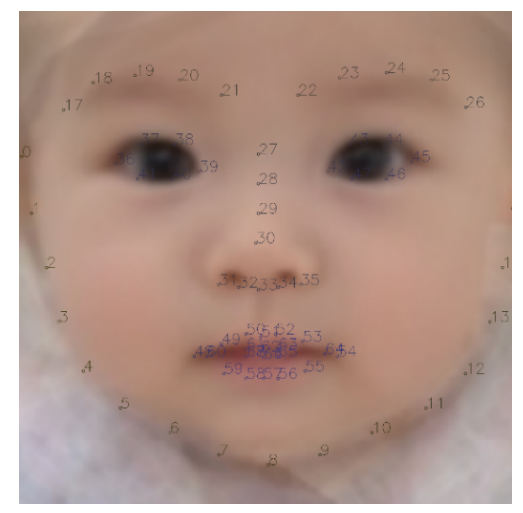

Figure 2: avarage baby face and lamdmark index

3.2.3 Inverse Age Progression. Inverse age progression is the process of generating a final image from the averaged age of the facial images at Get Face Landmark Index. This is all done without collapsing baby face images obtained in Average Baby Face Generation. The implementation uses a Dlib and OpenCV. It obtains feature points obtained by Dlib, performs image generation by passing the coordinate data of the feature points OpenCV side. Dlib automatically sorts the feature points obtained and treated them as a landmark. Its index is unchanged in all of the facial images. By setting the contribution ratio with respect to the index number, it is possible to process each selective parts. The color components are produced by Average Baby Face Generation, the present process is a concept that only features the specified parts inherited. If carried out well as deformation of 3D modeling, contour around the chin and other aspects should also be considered. The generated facial image is used by Live2D.

\subsection{Display of Haptics Synchronized with Voice}

When holding the baby doll, the doll is strengthening the interaction by vibro of Vibro transducer Vp2 (Vp210) which was mounted on the baby doll. This vibro is controlled by voice. Only by passing the band-pass filter using a Fourier transform effective frequency band $(5 \mathrm{~Hz} 200 \mathrm{~Hz})$ and extracted as the vibration data onto an output audio can it perform real-time tactile presentation through HMD attendant controller (Vive Controller). However, since it is the only vibrator that is built in the standard Vive controller, we felt its electric current output which vibrates the baby doll was insufficient after many exhibitions. To fix this problem we mounted the Vp210 onto Vive controller to improve the oscillating capability.

\section{IMPLEMENTATION OF IVRC 2016}

\subsection{Content of Experience}

This work allows for single player mode and two player collaboration. By making the photo of the players look younger and 
then combine the photographs together, the baby's face was born. Through taking care of the generated baby, players can experience the importance of parental love and family ties in this VR project (fig.3). The Vive controller resembles a milk bottle for the baby. Sensory clues such as the increase in milk consumption by the baby and a sense of touch are all presented. After the experience, a copy of the mother's pocket book contains the image of the baby's face was issued to the player as a souvenir.

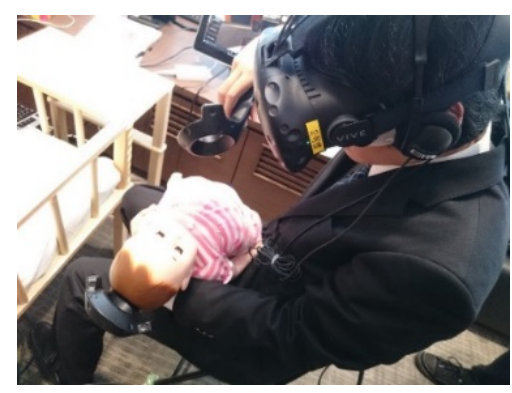

Figure 3: Picture of experience

\subsection{Real-time facial expression change of baby}

Fig. 4 is a block diagram of the system for generating a baby's face textures in real time. The essence of this work is to generate a texture of Baby face that changes according to the players. Because of this there is a major difference between our achieved research result and the below commonly used animation methods: Using the frame by frame animation technique that animates objects using subtle difference between each images and changing facial expression by changing the location of vertices of 3D models. Therefore, We implemented real-time facial expression changes by the use of RenderTexture which is one of Live2D and Unity features. In preparing vertex deformation data for use in Unity In Live2D, it was prepared as follows: Material texture to be used as the face of the template players are using (right eye, left eye, mouth, and foundation). Read a file that Live2DManager was generated by the inverse age progression first in Unity side, to draw using a method called the Graphics class DrawMeshNow. The texture was then rendered using a camera component drawing that have been Live2DModelUnity. Finally, the rendered texture was used to set the material of the face of the baby 3D model to the texture. Baby model state (cry, drink milk, sleep) is monitored by BabyEmotion to manipulate the Animator of animation state. Furthermore, in the Animator animation state was operated by parameters of Live2D, then the facial expression was changed. Live2DModelUnity's drawing, the rendering of RenderTexture, a Unity Reference, can change the texture in real time because it is performed every frame. It is generating the visual texture of baby face image in real time by the above operation.

\subsection{Interaction using image, voice and haptic}

Fig5 is a diagram that presents baby voices and vibro tactile associated with it. BabyEmotion reads wave files such as a baby's voice, which was prepared in advance from the audio folder, including: moody, laugh, cry, start drinking, stop drinking, waiting, and sleeping. This BabyEmotion sends the audio files corresponding to the parameter information to AudioSource where the audio file is outputted to a headphone via the HMD as the audio information is presented as haptic. Sampling period in this case is the same as drawing updated frequency (or 100 FPS). Vibration data is what tells TriggerHapticPulse how much should the ViveController vibrate. TriggerHapticPulse is a function within SteamVR_Controller which in term is a function within SteamVR_Plugin. This makes the vibrator controllable by Vive controller via the speech waveform. In parallel with the process of the spectrum of the voice data are WaveGetVibration and SteamVR_Plugin located inside Unity. In order to vibrate the controller mounted onto baby, WaveGetVibration uses SteamVR_Controller. After that, it gives the spectrum extracted from the above-mentioned audio data to the argument durationMicroSec of TriggerHapticPulse which is a method of SteamVR_Controller. The above processing is performed at the same frequency as the drawing update. In addition, while performing a process of the spectra of audio data, the process of giving the spectrum to the controller was at the same sampling period. Accordingly, the vibration waveform is to be reproduced from speech waveform and Vive controller to be reproduced from the headphones, approximately becomes the same waveform. On the other hand, the parameter information of BabyEmotion (are drinking milk, milk strikes in addition to mouth) is the event the baby is utteied in response to.

\section{RESULTS OBTAINED FROM EXHIBITION}

\subsection{Evaluation for current baby generation}

Although this system is capable of creating a baby's facial image that is similar to the players', in the questionnaire, which recorded the responses of 12 people comprised of both game developers and students, with "similar" on one end, "not similare" on the other end, and were asked to evaluate in four stages with the maximum score being 4 points symbolizing most similar. The average score was 2.27 points which can still be improved. Furthermore, it is necessary to generate a baby's facial image of different races especially for international exhibitions in the future.

\subsection{Haptic Feedback}

Compared to the standard installed vibrator Vive controller, although the Vive controller fitted with a Vp210 gives out a stronger vibration, if players shift their positions or change their postures while holding the baby, our system will have a hard time adjusting to the change. For future challenges we plan on enhancing the baby mockup.

\subsection{Possibility of Nursing Simulator using VR}

In recent years, the nursing educations in Japan, in order to foster nurses with practical skills that can support a diverse of clinical practices, are looking into simulation educational systems such as the objective structured clinical examination (OSCE). The practical simulator nursing education using virtual patients has attracted much attention. Our Virtual Reality work, "Real Baby - Real Family", as hinted by its name, was implemented using a realistic baby and is accessible to the general public where all can experience the love of a new founded family. This type of VR project remains unrealized 


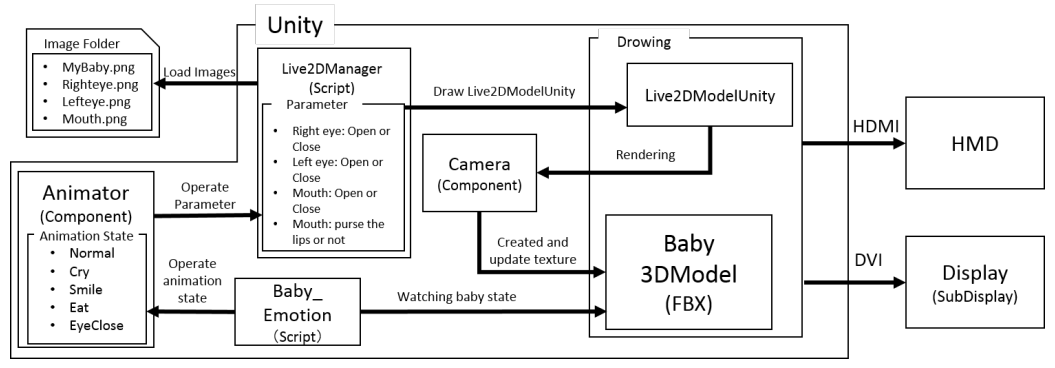

Figure 4: System of baby emotion

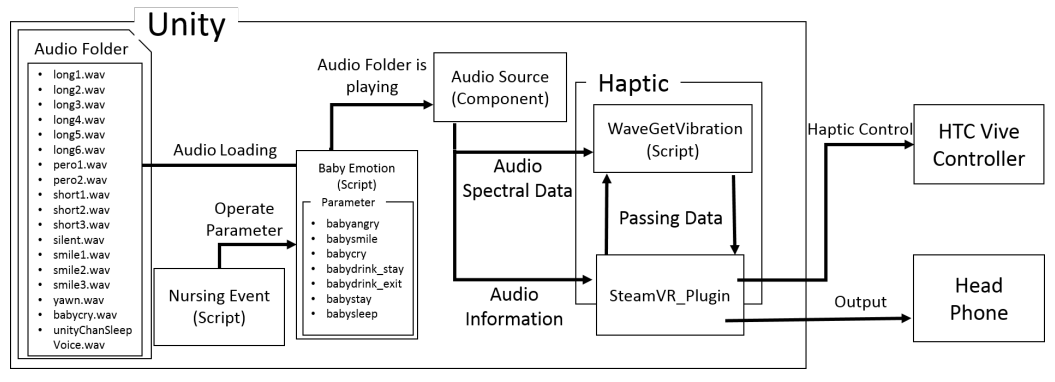

Figure 5: System of haptic by sounds

until now where we constructed a virtual baby that inherits facial features of the players, have a tactile sensation, and allows new and inexperienced parents to quickly become accustomed with raising a child. For future challenges, we hope to simulate other essential actions relating to raising a child such as breast feeding as well as giving the virtual baby a heartbeat and body temperature.

\subsection{Application to Entertainment System}

When exhibited at IVRC 2016, this VR system has an option that allows players to create a two-dimensional image of the baby from actual photographs of the players or substitution photos. This Virtual Reality system gives the illusion that the players really have created a baby whether the players are friends, heterosexual couples, or homosexual couples. The believable 3D baby model inside the virtual world and the $2 \mathrm{D}$ baby photo created from the players' image further enhances the experience. In the future we would like to tailor this entertainment system to the subculture market that makes two-dimensional images and babies.

\section{CONCLUSION}

IVRC 2016 Throughout the Tokyo qualifying and later the final competition, we continued to refine our system until it delivers a satisfying baby themed experience. Using the knowledge we gained from exhibitions, we adjusted the weight and vibration of the baby doll to produce a believable sense of touch when hugging the baby. Furthermore, we succeeded in creating a baby face that borrows features from the players' photographs and created a virtual baby capable of fostering deeper connections with the players than previous works such as YOTARO. We also experimented with a nursing simulator child care technique in this project and the result showed the potential of a nursing simulator technique for VR system. In the future we are hoping to create more VR entertainment systems featuring a complete VR world and real life physical objects such as $2 \mathrm{D}$ picture and baby doll.

Acknowledgement: Special thanks to Fujitsu Social Science Laboratory for helping us realizing this project.

\section{REFERENCES}

[1] Laerdal Medical Corporation. 1960. "SimNewB". (1960). http://www. kanazawa-med.ac.jp/ csc/pdf/no10.pdf.

[2] Ai Hasegawa. 2015. "(Im)possible_baby". (2015). http://aihasegawa.info/?works= impossible-baby-case-01-asako-moriga.

[3] Junichi Ido, Etsuko Ueda, Yoshio Matsumoto, and Tsukasa Ogasawara. 2006. 2P2-C20 Robotic Telecommunication System based on Facial Information Measurement. 7SME annual Conference on Robotics and Mechatronics (Robomec) (2006). http://ci.nii.ac.jp/naid/110008694348/

[4] Katunori Isono, Takayuki Hashimoto, and Masakazu Hori. 2004. Facial Aging Simulation Based on Facial Domain Knowledge. Computer Vision And Image Media 45, 8 (jun 2004), 10-14. http://ci.nii.ac.jp/naid/110002712413/

[5] H. Kozima. 2001. A Robot that Learns to Communicate with Human Caregivers. Proceedings of the First International Workshop on Epigenetic Robotics, 2001 (2001). http://ci.nii.ac.jp/naid/10014858498/

[6] Giorgio Metta, Giulio Sandini, Lorenzo Natale, and Francesco Panerai. 2001. Sensorimotor interaction in a developing robot.

[7] Yuya Mochizuki and Tatshya Nishikizawa. 2016. "Real baby / Real family". (2016). http://ivrc.net/2016/.

[8] Chiyoko Ono, Hiroki Kunimura, Madoka Hirai, Tetsuya Wagner Matsuzaki, Masatada Muramoto, Toshiaki Uchiyama, Kazuhito Shiratori, and Junichi Hoshino. 2009. Design Consideration in Developing Baby Type Robot. Bulletin of Japanese Society for Science of Design 56 (2009). http://ci.nii.ac.jp/naid/ 110007380346/

[9] B. Scassellati. 2001. Theory of mind for a humanoid robot. Autonomous Robots (2001). https://doi.org/10.1023/A:1013298507114

[10] Naoko Tosa. 1995. Neuro Baby. Journal of the Society of Instrument and Control Engineers (1995). http://ci.nii.ac.jp/naid/10006207702/ 